\title{
Phase behavior of a simple dipolar fluid under shear flow in an electric field
}

J. Liam McWhirter

Citation: The Journal of Chemical Physics 128, 034502 (2008); doi: 10.1063/1.2819485

View online: https://doi.org/10.1063/1.2819485

View Table of Contents: http://aip.scitation.org/toc/jcp/128/3

Published by the American Institute of Physics

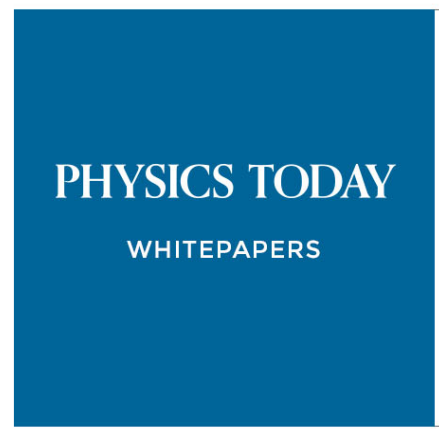




\title{
Phase behavior of a simple dipolar fluid under shear flow in an electric field
}

\author{
J. Liam McWhirter ${ }^{\text {a) }}$ \\ Institut für Festkörperforschung, Forschungszentrum Jülich, 52425 Jülich, Germany
}

(Received 7 September 2007; accepted 6 November 2007; published online 15 January 2008)

\begin{abstract}
Nonequilibrium molecular dynamics simulations are performed on a dense simple dipolar fluid under a planar Couette shear flow. Shear generates heat, which is removed by thermostatting terms added to the equations of motion of the fluid particles. The spatial structure of simple fluids at high shear rates is known to depend strongly on the thermostatting mechanism chosen. Kinetic thermostats are either biased or unbiased: biased thermostats neglect the existence of secondary flows that appear at high shear rates superimposed upon the linear velocity profile of the fluid. Simulations that employ a biased thermostat produce a string phase where particles align in strings with hexagonal symmetry along the direction of the flow. This phase is known to be a simulation artifact of biased thermostatting, and has not been observed by experiments on colloidal suspensions under shear flow. In this paper, we investigate the possibility of using a suitably directed electric field, which is coupled to the dipole moments of the fluid particles, to stabilize the string phase. We explore several thermostatting mechanisms where either the kinetic or configurational fluid degrees of freedom are thermostated. Some of these mechanisms do not yield a string phase, but rather a shear-thickening phase; in this case, we find the influence of the dipolar interactions and external field on the packing structure, and in turn their influence on the shear viscosity at the onset of this shear-thickening regime. (C) 2008 American Institute of Physics. [DOI: 10.1063/1.2819485]
\end{abstract}

\section{INTRODUCTION}

Under shear flow, the packing structure of particles around a specific, tagged particle in a fluid is distorted: the first solvation layer around the tagged particle ceases to be spherical under flow. ${ }^{1-8}$ For liquid composed of only molecules with a diameter at most on the order of 1 nanometer, the shear rates needed to obtain a significant distortion in the packing structure are impossible to generate experimentally; however, accessible shear rates are easily possible for a colloidal suspension where the suspended particles have diameters on the order of 100 nanometers to micrometers. ${ }^{9-13}$ In experiments the fluid packing structure can be measured by small angle neutron scattering (SANS) $;^{14,15}$ in simulations this structure is determined by calculating the pair distribution function. ${ }^{16}$

Nonequilibrium molecular dynamics (NEMD) methods have been created to simulate fluids under flow. ${ }^{16,17} \mathrm{~A}$ popular method has been the homogeneous SLLOD algorithm: in the case of a planar Couette flow, a constant shear is generated uniformly through the fluid by adding fictitious forces to the particle equations of motion complemented by LeesEdward boundary conditions. ${ }^{18}$ Heat generated by the flow must be extracted by a thermostat. Traditionally in NEMD, heat was extracted using kinetic thermostats, which kept the kinetic energy fixed at a target value or closely fluctuating about this value. The difficulty with such a thermostatting scheme was the determination of the flow profile or mean velocity profile of the fluid, which would then be subtracted

${ }^{a)}$ Electronic mail: j.mcwhirter@fz-juelich.de. from the total velocity of the fluid particles to give the thermal/peculiar velocity of these particles. ${ }^{19-25}$ For a planar Couette shear flow, there is no difficulty at low shear rates: the flow profile is simply linear where the shear gradient is just the shear rate inputted into the particle equations of motion. However, at very high shear rates, where the linear flow profile is no longer stable, thermostatting is made difficult by the need to determine the secondary flows that contribute, along with the linear flow, to the mean velocity profile. ${ }^{19}$ Recently, configurational thermostats ${ }^{26-31}$ have been devised that keep the configurational temperature ${ }^{32-34}$ fluctuating about a desired target value. These thermostats have a computational advantage over kinetic thermostats: configuration thermostats do not require knowledge of the flow profile; therefore, no decision has to be made over what method must be used to calculate the secondary flow profiles.

For a simple fluid composed of particles that interact through simple repulsive or Lennard-Jones pairwise interactions, NEMD simulations of a planar Couette flow at very high shear rates give very different fluid spatial structures depending on the thermostatting scheme chosen. Kinetic thermostats can be divided into two categories: profile biased and profile unbiased. ${ }^{19-22}$ In a planar Couette flow, profilebiased thermostats assume that secondary flows do not develop at any shear rate, that is, the flow profile is always linear; by contrast, profile-unbiased thermostats assume that secondary flows can develop at very high shear rates. Profilebiased NEMD simulations produce the string phase at high shear rates where particles are organized into strings aligned with hexagonal symmetry along the flow direction; ${ }^{7,8,35}$ this phase is characterized by a rapidly decreasing shear viscosity 
with increasing shear rate. However, profile-unbiased simulations produce a shear-thickening phase where the some particles organize into clusters roughly aligned along the compression axis of the flow; ${ }^{20,21,36,37}$ this phase is characterized by an increasing shear viscosity with increasing shear rate. Both these phases only occur once the shear rate becomes larger than some critical value. The string phase has never been clearly observed from experiments performed on a colloidal suspension; ${ }^{9-11}$ in addition, NEMD simulations using a configurational thermostated dynamics have produced the shear-thickening phase. ${ }^{26,28,37,38}$ Therefore, there is good reason to now believe that in the colloidal regime the string phase is a simulation artifact.

In this paper, we try to determine whether the string phase can be stabilized (brought into existence) by an externally applied electrical field. To couple this field to fluid particle motions, we insert into each particle a perfect dipole moment, ${ }^{36} \boldsymbol{\mu}$. Our fluid thus becomes a simple model for a colloidal suspension of ferromagnetic particles; ${ }^{39-41}$ however, we have neglected the hydrodynamic effects of a suspending solvent in our simulations. ${ }^{12,42}$ Of course, whether we obtain a string phase or a shear-thickening phase depends on our choice of thermostat. For simulations that do not yield the string phase, we will determine the influence of this field and the dipolar interactions on the packing structure of the fluid, and in turn their influence on the shear viscosity. We compare results obtained using different kinetic thermostated dynamics: the profile biased and unbiased dynamics mentioned above, as well as a more recent "extended" SLLOD dynamics devised by Bagchi and co-workers. ${ }^{43}$ We have also performed simulations using a configurational thermostated SLLOD dynamics for shear flow introduced by Delhommelle and co-workers; ${ }^{26}$ we will briefly note some results of our preliminary investigation using this thermostat.

As mentioned above, our dipolar fluid is a simple model of a magnetic ferrofluid, a stable colloidal suspension of single-domain ferromagnetic particles. ${ }^{39,40}$ Each particle in such a suspension is coated with adsorbed long-chain molecular species on the particle surface to prevent agglomeration of the particles to each other. An example that has been used in one experiment consists of iron nitride core particles in kerosene ${ }^{41}$ another common experimental example consists of magnetite $\left(\mathrm{Fe}_{3} \mathrm{O}_{4}\right)$ core particles. Characterizing the magnetoviscous properties of these suspensions under fluid flow has attracted much interest: that is, the determination of the viscosity tensor relating the stress tensor to the strain rate tensor for a ferrofluid subjected to an external magnetic field. ${ }^{44,45}$ The viscous response depends on the magnitude of the applied field as well as its orientation relative to the flow geometry. The first response theory was constructed for an infinitely dilute suspension and neglected thermal fluctuations, ${ }^{46}$ and compared well with experimental results obtained by McTague. ${ }^{47}$ Since then, kinetic theories for dilute ferromagnetic fluids consisting of spheres, dumbbells, and rods have been developed that include thermal effects. ${ }^{44,45,48,49}$ A qualitative explanation of the response for dilute suspensions is that the application of an external magnetic field hinders the rotation of the ferromagnetic particles in a solvent velocity gradient, which results in the rotation of the particle being different than the vorticity of the background solvent flow; this difference creates an additional dissipation of heat. ${ }^{46,47}$ For spherical particles, the effective shear viscosity (or Miesowicz viscosity) for a planar Couette flow will be greater if the uniform external field is directed parallel to the flow than if this field is parallel to the mean velocity or flow gradient direction. For axisymmetric particles like rods, the opposite is true: the viscosity is greater when the external field is parallel to the flow gradient. ${ }^{45}$ With increasing the number density, one cannot neglect dipoledipole interactions between the particles; ${ }^{49,50}$ for example, in nondilute systems with sufficiently high single-particle dipole moments, chains of dipoles, oriented head to tail, can form. As with a suspension of dipolar rods, the shear viscosity given the existence of chains will be greater if the external field is directed parallel to the flow gradient. ${ }^{49}$ With increasing the number density, hydrodynamic (solvent flow) mediated interactions between different particles become relevant to the particle dynamics; at high number densities (packing fractions between 0.1 and 0.4 ), flow-induced distortions to the fluid's spatial microstructure should also be incorporated into any theory of the magnetoviscous response. Recent theoretical efforts have started to extend kinetic theory to account for dipole-dipole and excluded volume interactions in a mean-field approximation $;{ }^{50}$ however, this effort has been restricted to the regime of small flow gradients and weakly interacting dipoles. By contrast, the simulations performed in this paper were done at the relatively high flow gradients and dipole moments. However, we do not attempt in this paper to characterize the full viscous response of our fluid; we only compare the nonequilibrium shear viscosity between a Lennard-Jones fluid, the corresponding simple dipolar fluid (a Stockmayer fluid), and this simple dipolar fluid where an external field (be it an electric or magnetic field) is directed along the vorticity direction, perpendicular to both the flow and flow gradient directions. Our packing fraction is very high, $\phi \sim 0.46$; we partially explain our simulation results based on the changes in the spatial packing structure of our fluid particles.

The remainder of this paper is organized as follows. In Sec. II, we present basic SLLOD dynamics applied to a planar Couette flow, describing the kinetic thermostated, profile-biased, and profile-unbiased dynamics. The fluid model and simulation details are then given in Sec. III. NEMD simulation results pertaining to profile-biased, profile-unbiased, and the extended SLLOD dynamics are discussed sequentially in Secs. IV A-IV C. The configurational thermostated SLLOD dynamics is presented in Sec. IV B since it produces qualitatively the same results as the kinetic thermostated, profile unbiased dynamics. Finally, in Sec. V, we summarize our work and discuss its possible merits.

\section{METHOD}

The translational motion of the fluid undergoing a planar Couette flow at a shear rate $\gamma$ is governed by SLLOD dynamics ${ }^{17,51}$ accompanied by a Gaussian thermostat,

$d \mathbf{r}_{i} / d t=\mathbf{p}_{i}^{\prime} / m+\hat{\mathbf{e}}_{x} y_{i} \gamma$, 


$$
d \mathbf{p}_{i}^{\prime} / d t=\mathbf{F}_{i}-\hat{\mathbf{e}}_{x} \gamma \mathbf{p}_{i y}^{\prime}-\alpha^{T}\left[\mathbf{p}_{i}^{\prime}-m \overline{\mathbf{u}}_{s}^{g}\left(\mathbf{r}_{i}, t\right)\right] .
$$

The Gaussian thermostat keeps the translational kinetic thermal energy of the fluid, $(1 / 2 m) \sum_{i}\left(\mathbf{p}^{\prime \prime}\right)^{2}=3(N-1) k_{B} T_{\text {kin,tr }} / 2$, constant where the translational kinetic temperature, $T_{\text {kin,tr }}$, is set to a desired target temperature $T$. This dynamics is accompanied by Lees-Edwards boundary conditions ${ }^{18}$ where the layer of image cells in the $Y$ direction above and below the central simulation cell move with velocities $\gamma L \hat{\mathbf{e}}_{x}$ and $-\gamma L \hat{\mathbf{e}}_{x}$. These cells are cubic with sides of length $L$ and volume $V$. Here, $\mathbf{F}_{i}$ is the total force on particle $i$ due to all the other particles. The total momentum is $\mathbf{p}_{i}=\mathbf{p}_{i}^{\prime}+\hat{\mathbf{e}}_{x} \gamma y_{i} m=\mathbf{p}_{i}^{\prime \prime}$ $+\overline{\mathbf{u}}_{s}^{g}\left(\mathbf{r}_{i}, t\right) m+\hat{\mathbf{e}}_{x} \gamma y_{i} m$, where $\overline{\mathbf{u}}_{s}^{g}(\mathbf{r}, t)$ is a "regularized" secondary flow ${ }^{51}$ and $\mathbf{p}_{i}^{\prime \prime} / m$ is the peculiar, "thermal" velocity. The streaming velocity (that is, the mean velocity profile) is $\overline{\mathbf{u}}\left(\mathbf{r}_{i}, t\right)=\overline{\mathbf{u}}_{s}^{g}\left(\mathbf{r}_{i}, t\right)+\hat{\mathbf{e}}_{x} \gamma y_{i}$. The secondary flow is related to the spatial average (over some small volume centered about position $\mathbf{r}$ ) of the instantaneous microscopic expression for the momentum density, ${ }^{51}$

$$
\mathbf{J}(\mathbf{r}, t)=\rho(\mathbf{r}, t) \mathbf{u}_{s}^{g}(\mathbf{r}, t)=\sum_{i} \mathbf{p}_{i}^{\prime}(t) \delta\left[\mathbf{r}-\mathbf{r}_{i}(t)\right] .
$$

In the case of profile biased dynamics, $\overline{\mathbf{u}}_{s}^{g}$ in Eq. (2) is set to zero. Setting $\overline{\mathbf{u}}_{s}^{g}$ to zero in Eq. (2) does not mean that the biased dynamics will not generate a secondary flow. Rather, if any secondary flow is generated, the Gaussian thermostatting term in Eq. (2) containing the multiplier $\alpha^{T}$ will produce an external force ${ }^{17,21,51}$ proportional to $\overline{\mathbf{u}}_{s}^{g}$ that will prevent the amplification of $\overline{\mathbf{u}}_{s}^{g}$. Simply put, any secondary flow is interpreted by the thermostat as heat and so is damped. In the case of profile unbiased dynamics, $\overline{\mathbf{u}}_{s}^{g}$ is not set to zero in Eq. (2): $\overline{\mathbf{u}}_{s}^{g}$ can be calculated by minimizing a residual that contains the $\mathbf{p}_{i}^{\prime}(t)$ and a truncated Fourier series representation for the secondary flow. ${ }^{21,22}$ Solving this minimization for the Fourier coefficients, given the truncation, is approximately equivalent to spatially averaging ${ }^{51}$ the instantaneous microscopic expression for $\mathbf{u}_{s}^{g}$ given by Eq. (3). This minimization yields a set of coupled linear equations for the coefficients which must be solved at every time step during a simulation.

The dipoles are uniaxial particles; they inherit a streaming angular velocity, ${ }^{21,23} \boldsymbol{\Lambda}_{i}^{b}=\mathbf{A}_{i} \cdot \boldsymbol{\Lambda}$, from the streaming translational velocity, $\gamma y \hat{\mathbf{e}}_{x}$. Here, $\mathbf{A}_{i}=\mathbf{A}\left(\boldsymbol{\Omega}_{i}\right)$ is the rotation matrix from the laboratory to the body-fixed frame of particle $i$, where $\boldsymbol{\Omega}_{i}$ is the orientation of particle/dipole $i$ in the laboratory frame. ${ }^{16}$ This streaming velocity must be treated when thermostatting the angular velocity or $\boldsymbol{\omega}$ dynamics in the body-fixed frame (denoted by a superscript $b$ ),

$$
I \dot{\boldsymbol{\omega}}_{i}^{b}=\mathbf{T}_{i}^{b}-\alpha^{R}\left(\boldsymbol{\omega}_{i}^{b}-\boldsymbol{\Lambda}_{i}^{b}\right)+\mathbf{T}_{E, i}^{b},
$$

where $\mathbf{T}_{E, i}^{b}$ is an external torque in the body-fixed frame due to an externally applied field. For an electric (or magnetic) field, $\mathbf{E}$, the torque in the laboratory frame is

$$
\mathbf{T}_{E, i}=\boldsymbol{\mu} \times \mathbf{E},
$$

where $\boldsymbol{\mu}$ is the dipole moment of a single particle and $\mathbf{T}_{E, i}^{b}$ $=\mathbf{A}_{i} \cdot \mathbf{T}_{E, i}$. For simplicity, in the Gaussian isokinetic thermostat with a multiplier, $\alpha^{R}$, we set $\boldsymbol{\Lambda}$ equal to the vorticity in the linear shear flow profile: $\boldsymbol{\Lambda}=(1 / 2) \nabla \times\left[\gamma y \hat{\mathbf{e}}_{x}\right]=$ $-(1 / 2) \gamma \hat{\mathbf{e}}_{z}$. More sophisticated choices for $\boldsymbol{\Lambda}$ exist, ${ }^{23}$ though in our experience no significant quantitative differences arise from using them for our model fluid under a planar Couette flow. ${ }^{36}$ The Gaussian thermostat keeps the orientational kinetic thermal energy of the fluid, $(1 / 2) I \Sigma_{i}^{N}\left[\boldsymbol{\omega}_{i}^{b}-\boldsymbol{\Lambda}_{i}^{b}\right]^{2}$ $=k_{B} T_{\text {kin,or }}$, constant where the orientational kinetic temperature, $T_{\text {kin,or }} / 2$, is set equal to a desired temperature $T$.

We choose to describe the orientation of the body-fixed coordinate frame with respect to the laboratory-fixed coordinate frame by quaternions, $\mathbf{q}=\left\{q_{l}=q_{l}(\boldsymbol{\Omega}) ; l=1,4\right\}$, which satisfy the constraint $\Sigma_{l} q_{l}^{2}=1$. Using quaternions has the advantage that divergences in the orientational equations of motion do not occur; ${ }^{16,52}$ these divergences are encountered when integrating the equations of motion for the Euler angles which alternatively can describe the orientation of a dipole in the space-fixed frame. The dynamics of the orientational degrees of freedom specified by quaternions is governed by $\dot{\mathbf{q}}_{i}=(1 / 2) \mathbf{W}\left(\mathbf{q}_{i}\right) \cdot \widetilde{\boldsymbol{\omega}}_{i}$. Here, $\widetilde{\boldsymbol{\omega}}_{i}$ is a column vector whose elements are $0, \omega_{x}^{b}, \omega_{y}^{b}$, and 0 going from top to bottom; $\mathbf{W}\left(\mathbf{q}_{i}\right)$ is a $4 \times 4$ matrix whose elements are specified in Ref. 16 .

\section{MODEL AND SIMULATION DETAILS}

We consider a Stockmayer fluid consisting of LJ particles each embedded at the center with a point dipole, $\boldsymbol{\mu}$. These dipolar particles interact through the pair potential, $u$ $=u_{\mathrm{LJ}}+u_{d d}$, where

$$
\begin{aligned}
& u_{\mathrm{LJ}}(r)=4 \epsilon\left[\left(\frac{\sigma}{r}\right)^{12}-\left(\frac{\sigma}{r}\right)^{6}\right], \\
& u_{d d}\left(\mathbf{r}_{i j}, \boldsymbol{\mu}_{i}, \boldsymbol{\mu}_{j}\right)=\frac{1}{4 \pi \xi}\left[\frac{\boldsymbol{\mu}_{i} \cdot \boldsymbol{\mu}_{j}}{r_{i j}^{3}}-\frac{3\left(\boldsymbol{\mu}_{i} \cdot \mathbf{r}_{i j}\right)\left(\boldsymbol{\mu}_{j} \cdot \mathbf{r}_{i j}\right)}{r_{i j}^{5}}\right] .
\end{aligned}
$$

Here, $r=|\mathbf{r}|$ is the interparticle separation, and $\xi$ is either the permittivity or permeability of the solvent depending on whether the $\boldsymbol{\mu}$ 's represent electric or magnetic dipole moments. The LJ pair potential was calculated using a minimum image convention with a spherical cutoff at $r=2.5 \sigma$. All quantities are expressed in terms of reduced units: number density, $\rho^{*}=\rho \sigma^{3}=0.88$ (packing fraction, $\phi=\rho^{*} \pi / 6$ ); temperature, $T^{*}=k_{B} T / \epsilon=1.125$; dipole moment, $\mu^{*}$ $=\mu /\left(4 \pi \xi \sigma^{3} \epsilon\right)^{1 / 2} ;$ moment of inertia, $I^{*}=I /\left(m \sigma^{2}\right)=0.025$; shear rate, $\gamma^{*}=\left(\sigma^{2} m / \epsilon\right)^{1 / 2} \gamma=0$ to 4.5 ; and time step, $(\Delta t)^{*}$ $=\left(\epsilon / \sigma^{2} m\right)^{1 / 2} \Delta t=0.0005$. For most simulations, the central simulation cell contained $N=256$ particles; however, when using the configurational thermostat, we performed simulations with $N=500$ and 1372 particles per cell (Sec. IV B). Henceforth, the asterisks are dropped; all variables are assumed reduced unless otherwise stated.

The long-range dipolar interactions are taken into account using the Ewald summation method ${ }^{53-55}$ adapted to Lees-Edwards boundary conditions. ${ }^{18,56}$ Explicit expressions for the energy and for the pressure are given in Ref. 36. Conducting boundary conditions were used in all simulations. For the simulations evolved using profile-biased and -unbiased dynamics, we examined a simple dipolar fluid with a single particle dipole moment of $|\boldsymbol{\mu}|=\mu=2$, and with $\mu$ $=2.3$. For $\mu=2$, the Ewald parameter $\alpha$ was set at 5.5, and terms up to $n_{\max }=5$ were included in the Fourier space sum; for $\mu=2.3$, we set $\alpha=6$ and $n_{\max }=6$. Configurational thermo- 


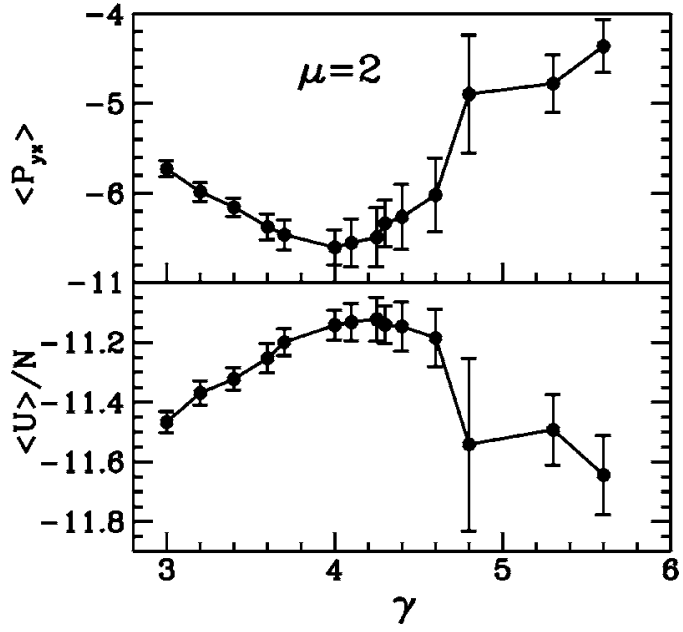

FIG. 1. Kinetic thermostated, profile-biased dynamics. Average potential energy, $\langle U\rangle / N$, (in units of $\epsilon$ ) and shear pressure, $P_{y x}$, as functions of shear rate $\gamma$ for the simple dipolar fluid with a single particle dipole moment of $\mu=2$. The electric field is off.

stated SLLOD and extended SLLOD dynamics simulations were performed on just the $\mu=2$ dipolar fluid.

Kinetic thermostated, biased, and unbiased dynamics simulations at $\gamma \neq 0$ were run for 400000 time steps of size $\Delta t=0.0005$, starting from an equilibrium configuration, that is, a configuration at $\gamma=0$. Averages were then collected during a subsequent 400000 time steps; standard deviations were estimated by dividing these into 20 blocks of 20000 . The configurational thermostated and extended SLLOD dynamics simulations were run slightly differently; details of these simulations are given below in Secs. IV B and IV C. All equations of motion were integrated using a fifth-order Gear predictor-corrector algorithm. ${ }^{16}$

\section{RESULTS}

\section{A. Profile-biased dynamics}

First, we consider simulation runs where the electric field is off, $E=0$. With $\mu=2$ and 2.3, the internal energy of these fluids, $\langle U\rangle / N$, increases while the shear viscosity, $\eta_{s}$ $=-P_{y x} / \gamma$, decreases for increasing $\gamma$, provided the $\gamma$ is less than some critical value of shear rate which we will call $\gamma_{\mathrm{cr}}^{\text {str }}$ (Figs. 1 and 2). These two trends can be related to the distortions in the fluid's spatial structure, which can be seen by measuring the increase in the anisotropy of the pair distribution function, $g(\mathbf{r})$, with increasing $\gamma$. At $\gamma_{\mathrm{cr}}^{\mathrm{str}}=4.15 \pm 0.15$ for $\mu=2$ and $\gamma_{\text {cr }}^{\text {str }}=4.85 \pm 0.15$ for $\mu=2.3$, the $\eta_{s}$ for both these fluids begins to rapidly decrease or drop with increasing $\gamma$; in addition, $\langle U\rangle / N$ begins to decrease with increasing $\gamma$. This rapid drop in $\eta_{s}$ reflects a drastic change in the spatial organization of the particles: the particles are organizing themselves into strings aligned along the direction of the flow (parallel to the $X$ axis) with hexagonal symmetry. The effective particle number density in the $Z$ direction (perpendicular to the flow and shear gradient directions) is larger than this density along the axis of a string, parallel to the direction of flow. ${ }^{7,836}$ With conducting (metallic) boundary conditions, the formation of this string phase is accompanied by a strong orientational ordering of the dipoles where the dipoles align

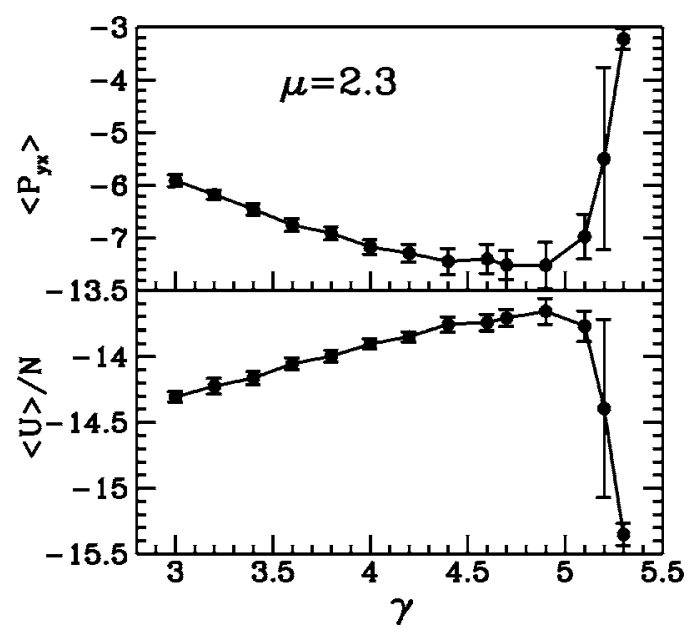

FIG. 2. Same caption as Fig. 1 except now $\mu=2.3$.

so that their symmetry axes are almost parallel to the $Z$ axis, along which the particle number density is relatively high. ${ }^{36}$ The orientational response, as measuring by the $P_{1}$ order parameter, occurs more quickly with increasing $\gamma$ at $\gamma \gg \gamma_{\mathrm{cr}}^{\mathrm{str}}(\mu)$ for the $\mu=2.3$ fluid than for the $\mu=2$ fluid (Fig. $3)$. This order parameter is defined by

$$
P_{1}=\frac{1}{N}\left|\sum_{i=1}^{N} \hat{\mathbf{u}}_{i} \cdot \hat{\mathbf{d}}\right|,
$$

where $\hat{\mathbf{u}}_{i}=\boldsymbol{\mu}_{i} / \mu$ is the unit vector parallel to the dipole in particle $i$, and $\hat{\mathbf{d}}$ is the unit eigenvector associated with the largest eigenvalue of the second rank order tensor, ${ }^{16,57}$

$$
\mathbf{O}=\frac{3}{2}\left[\frac{1}{N} \sum_{i=1}^{N} \hat{\mathbf{u}}_{i} \hat{\mathbf{u}}_{i}-\frac{1}{3} \mathbf{1}\right] .
$$

Presumably, as the dipole moment, $\mu$, becomes larger, the dipole's orientation becomes more sensitive to changes in the spatial correlations within the fluid. The critical shear rate also increases with increasing $\mu$ : increasing $\mu$ has the effect

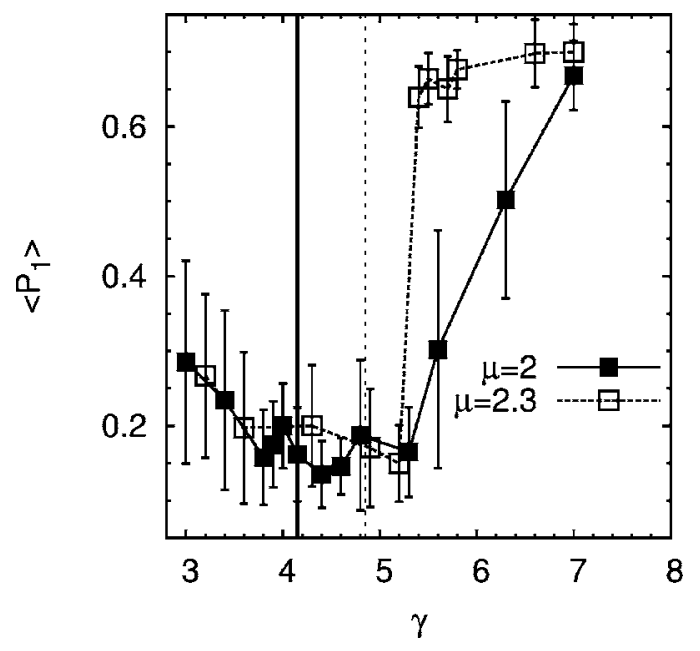

FIG. 3. Kinetic thermostated, profile-biased dynamics. $P_{1}$ order parameter as a function of shear rate $\gamma$ for the simple dipolar fluids with $\mu=2$ and 2.3. Vertical lines mark critical value of shear rate, $\gamma_{\mathrm{cr}}^{\mathrm{str}}$, where the shear viscosity, $\eta_{s}=-P_{y x} / \gamma$, first begins to decrease rapidly with increasing $\gamma$. The electric field is turned off. 


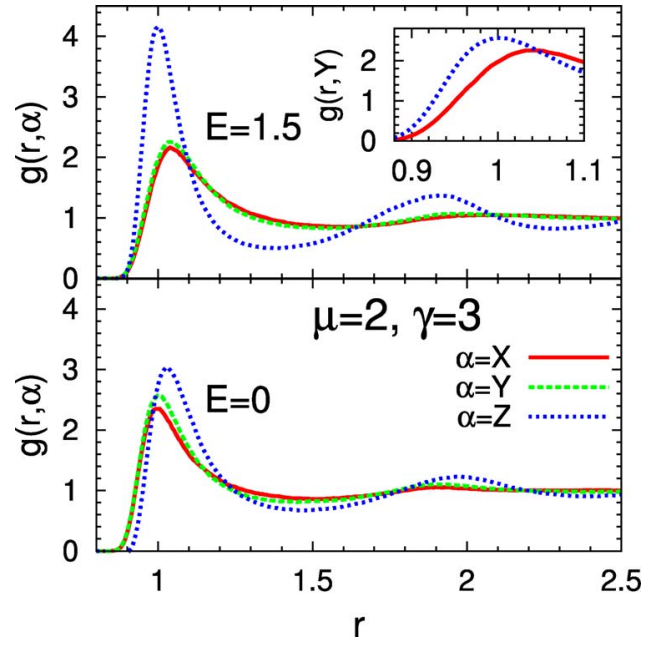

FIG. 4. (Color online) Kinetic thermostated, profile-biased dynamics. Pair distribution function, $g(r, \alpha)$, resolved along the $X$ (solid), $Y$ (dashed), and $Z$ (dotted) directions where $X$ and $Y$ are the flow and shear gradient directions, respectively. The single particle dipole moment is $\mu=2$, and the shear rate is $\gamma=3<\gamma_{\mathrm{cr}}^{\mathrm{str}}(E)$ at $E=0$ (bottom panel, no electric field) and $E=1.5$ (top panel). The inset in the top panel gives the pair distribution function along the $Y$ direction for $E=0$ (dotted curve in inset) and $E=1.5$ (solid curve in inset).

of increasing the effective attractions between particles; since the fluid's spatial structure is elongated along the direction of the flow during the formation of the strings, this increased attraction will resist this elongation, thereby shifting $\gamma_{\mathrm{cr}}^{\mathrm{str}}$ to higher values.

We now consider simulation runs where the electric field is turned on, $E \neq 0$, and compare the results here with those from runs where the field is turned off, $E=0$. The electric field is parallel to the $Z$ direction, perpendicular to the flow and the shear gradient directions. As shown in Fig. 4, this field at $\gamma<\gamma_{\mathrm{cr}}^{\text {str }}$ has the effect of increasing the effective particle density along the $Z$ axis; to compensate for this increase, the density along the $X$ and $Y$ directions is decreased. As a result, there is less friction between adjacent fluid planes that are parallel to the $Y Z$ plane, and the shear viscosity decreases with increasing $E$ (Fig. 5). Comparing the $E$ $\neq 0$ to the $E=0$ results, we also see from Fig. 5 that the $\gamma_{c r}^{\text {str }}$ decreases with increasing $E$. The particle density along the $Z$ direction increases dramatically in going from $\gamma<\gamma_{\text {cr }}^{\text {str }}$ to $\gamma$ $>\gamma_{\text {cr }}^{\text {str }}$ (Fig. 6). By applying an electric field parallel to the $Z$ axis, we externally bias the fluid toward a density distribution that is more consistent with the density distribution of a string phase. Prior to performing the simulations, we believed, based on physical intuition, that this bias from the electric field $(E \neq 0)$ on the simple dipolar fluid $(\mu=2$ and 2.3) would result in the transition to the string phase beginning at a lower value of $\gamma_{\mathrm{cr}}^{\mathrm{str}}$ : if a radical deformation in spatial structure with increasing $\gamma$ (as happens during the formation of a string phase) at $E=0$ gives rise to a strong orientational ordering, then maybe the converse is true; a strong orientational ordering induced by an external field $(E \neq 0)$ can give rise to a radical deformation in spatial structure with increasing $\gamma$ starting at a $\gamma<\gamma_{\mathrm{cr}}^{\mathrm{str}}(E=0)$.

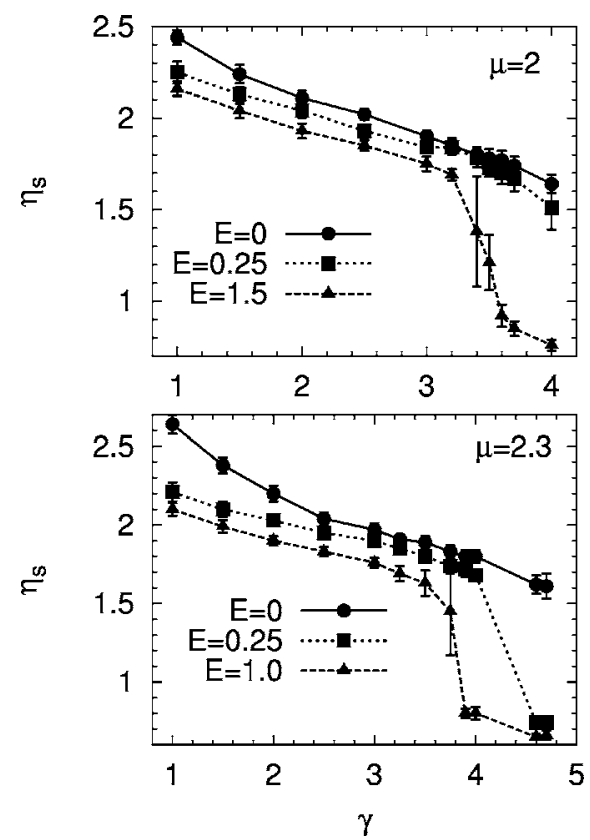

FIG. 5. Kinetic thermostated, profile-biased dynamics. Shear viscosity, $\eta_{s}$ $=-P_{y x} / \gamma$, as a function of shear rate $\gamma$ for the simple dipolar fluids with a single particle dipole moment of $\mu=2$ (top panel) and $\mu=2.3$ (bottom panel). The electric field intensity, $E$, is varied.

\section{B. Profile-unbiased dynamics}

In the previous section, we demonstrated that if the string phase exists, then the transition to this phase can begin at a smaller critical shear rate if an electric field directed along the $Z$ axis is applied to the simple dipolar fluid. However, the existence of the string phase in simulations is known to depend on the choice of the thermostat used. Kinetic thermostated, profile-unbiased dynamics, where the secondary flow $\overline{\mathbf{u}}_{s}^{g}$ is set to zero in Eq. (2), yields no string phase at high shear rates. ${ }^{19,21,36,51}$ Figure 7 confirms this finding; in addition, the string phase is not stabilized for the

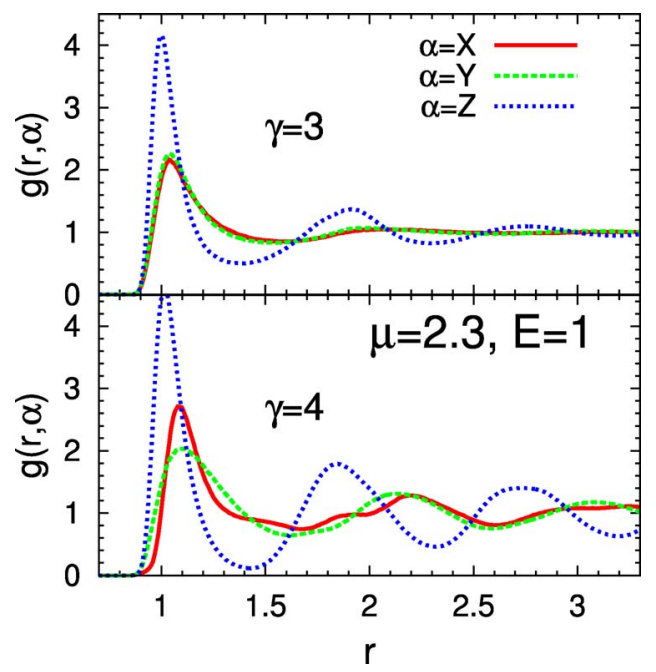

FIG. 6. (Color online) Kinetic thermostated, profile-biased dynamics. Pair distribution function, $g(r, \alpha)$, resolved along the $X$ (solid), $Y$ (dashed), and $Z$ (dotted) directions. The single particle dipole moment is $\mu=2.3$, and the electric field intensity is $E=1$. For the top panel the shear rate is $\gamma=3$ $<\gamma_{\mathrm{cr}}^{\mathrm{str}}=3.75 \pm 0.15$; for the bottom panel $\gamma=4>\gamma_{\mathrm{cr}}^{\mathrm{str}}$ at these values of $\mu$ and E. 


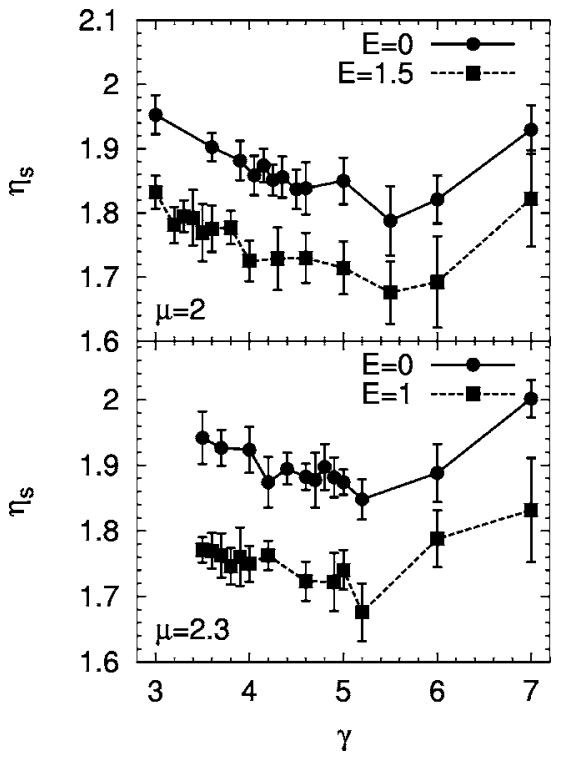

FIG. 7. Kinetic thermostated, profile unbiased dynamics. Shear viscosity, $\eta_{s}=-P_{y x} / \gamma$, as a function of shear rate, $\gamma$, for a simple dipolar fluid with $\mu=2$ (top panel) and 2.3 (bottom panel). The electric field intensity, $E$, is varied.

simple dipolar fluid with $\mu=2$ or 2.3 by the application of an electric field parallel to the $Z$ axis. The electric field, however, still reduces the shear viscosity, $\eta_{s}$, of these fluids.

Instead of the shear-thinning phase at high shear rates where the shear viscosity increases rapidly with increasing shear rate, we obtain a phase that undergoes shear thickening: $\eta_{s}$ increases with increasing $\gamma$ for those $\gamma^{\prime}$ 's greater than a new critical shear rate, $\gamma_{\mathrm{cr}}^{\text {th }}$. Comparing the results shown in Fig. 7 to those obtained using a profile-biased dynamics, we find that the onset of shear thickening occurs at a higher shear rate than the critical shear rate, marking the start of the transition to a string phase: $\gamma_{\mathrm{cr}}^{\mathrm{th}}>\gamma_{\mathrm{cr}}^{\mathrm{str}}$. Furthermore, $\gamma_{\mathrm{cr}}^{\mathrm{th}}$ appears relatively independent of the magnitude of the electric field $\left[\gamma_{\mathrm{cr}}^{\text {th }}(E=0) \sim \gamma_{\mathrm{cr}}^{\text {th }}(E \neq 0)\right]$ and the dipole moment $\left[\gamma_{\mathrm{cr}}^{\text {th }}(\mu\right.$ $\left.=2) \sim \gamma_{\mathrm{cr}}^{\mathrm{th}}(\mu=2.3)\right]$, at least for the values of $E$ and $\mu$ that we have considered.

Using a kinetic thermostat where only the kinetic degrees of freedom are thermostated is not the only way to achieve an unbiased dynamics. One can alternatively choose to thermostat the configurational, not the kinetic, degrees of freedom. ${ }^{26,31}$ Delhommelle, Petravic, and Evans have devised a version of SLLOD dynamics where only the translational configurational degrees of freedom are thermostated, ${ }^{26}$

$$
\begin{aligned}
& \frac{d \mathbf{r}_{i}}{d t}=\frac{\mathbf{p}_{i}^{\prime}}{m}+\hat{\mathbf{e}}_{x} y_{i} \gamma+\frac{s}{T} \frac{\partial T_{\mathrm{conf}, \mathrm{tr}}^{I}}{\partial \mathbf{r}_{i}}, \\
& \frac{d \mathbf{p}_{i}^{\prime}}{d t}=\mathbf{F}_{i}-\hat{\mathbf{e}}_{x} \gamma \mathbf{p}_{i y}^{\prime}, \\
& \dot{s}=-Q_{c} \frac{\left(T_{\text {conf }, \mathrm{tr}}^{I}-T\right)}{T} .
\end{aligned}
$$

Here, $T_{\text {conf,tr }}^{I}$ is the instantaneous translational configurational temperature, ${ }^{33}$ while $T$ is the desired "target" temperature. This thermostated dynamics uses a feedback mechanism similar to a Nosé-Hoover mechanism, Eq. (12) with control parameter $Q$, to keep $T_{\text {conf,tr }}^{I}$ fluctuating close to $T$. The advantage of using a configurational thermostat is that the secondary flows are not required in the equations of motion in order to evolve the system forward in time; however, when the linear flow profile ceases to be stable, the configurational thermostat does not affect the evolution of the secondary flows, that is, these flows are allowed to develop. ${ }^{26}$ Braga and Travis have devised two other configurational thermostats $^{31}$ that generate the canonical ensemble at equilibrium, $\gamma=0$, by using a Nosé-Hoover feedback mechanism. Equations (10)-(12) have not been shown mathematically to constitute a Nosé-Hoover type of dynamics; therefore, no formal proof has been presented that these equations will also generate a canonical ensemble, despite their ability to keep $T_{\text {conf,tr }}^{I}$ fluctuating closely about a target temperature. The canonical ensemble is not the steady-state ensemble for a nonequilibrium simple fluid under shear flow; in fact, the mathematical form for the nonequilibrium ensemble is not known for this fluid, so Monte Carlo simulations of a simple fluid under shear flow cannot be performed that reproduce NEMD simulation results. ${ }^{58}$ However, it does seem sensible to choose a nonequilibrium dynamics that extrapolates to a correct equilibrium dynamics in the limit of the driving thermodynamic force going to zero. The configurational thermostats derived by Braga and Travis also have the practical advantage that they can keep the configurational temperature of the fluid close to the target even when the target undergoes a sudden change in value; by contrast, Eqs. (10)-(12) are unable to perform this task, and so are much less robust. The dynamics devised by Delhommelle and co-workers has performed very well as a thermostat in many simulation studies; as a result, despite its conceptual and practical drawbacks, we have chosen to use this dynamics in our present, preliminary study. A more systematic study of a simple dipolar fluid at high shear rates that compares results using various different configurational thermostats is forthcoming.

The microscopic expression for $T_{\text {conf,tr }}$ was derived from the thermodynamic relation $1 / T=(\partial S / \partial U)_{V}$, where averages $\langle\cdot\rangle$ over phase space are weighted by equilibrium statistical ensembles, ${ }^{32,33}$

$$
\frac{1}{T_{\text {conf,tr }}}=\left\langle\frac{\sum_{i} \nabla_{\mathbf{r}_{i}}^{2} U}{\sum_{j}\left|\nabla_{\mathbf{r}_{j}} U\right|^{2}}\right\rangle=\left\langle\frac{1}{T_{\text {conf,tr }}^{I}}\right\rangle,
$$

where $U$ is the potential energy of the fluid. Strictly speaking, this expression should only be used at equilibrium where no flow (mean velocity) gradients exist, or at least in nonequilibrium situations where flow gradients measured across several particle diameters are sufficiently negligible that the local equilibrium assumption of linear irreversible thermodynamics can be invoked. Our simulations are performed far away from equilibrium at very high shear rates where there are significant distortions in the spatial packing structure of the model fluid. Since the exact nonequilibrium statistical mechanical ensemble for a simple fluid under shear flow is not known, ${ }^{58}$ the exact microscopic definition for the configurational temperature at nonequilibrium cannot be derived. Therefore, using the equilibrium microscopic expres- 


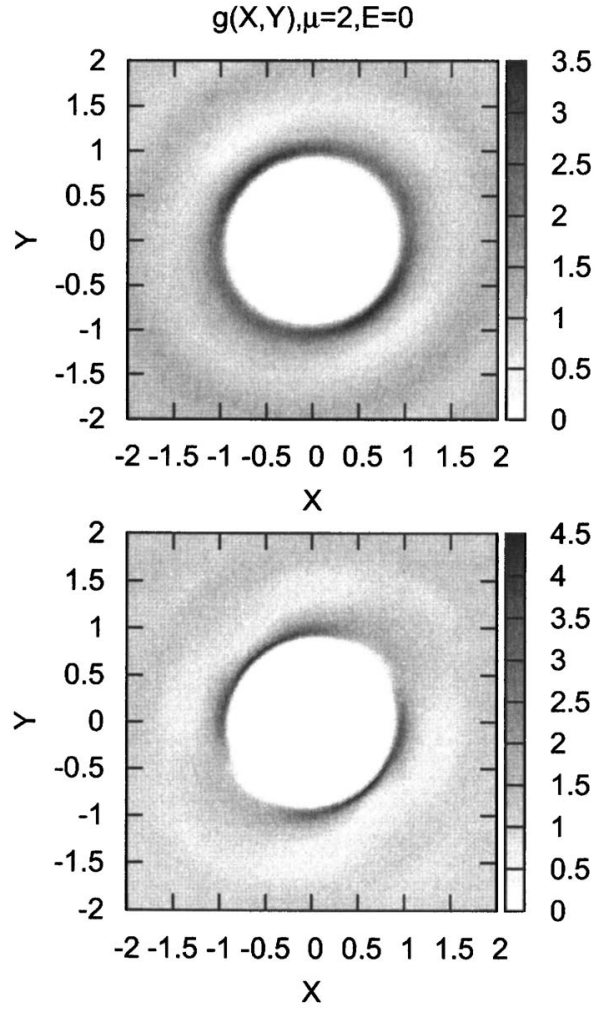

FIG. 8. Configurational thermostated SLLOD dynamics. Pair distribution function in the $X Y$ plane for the simple dipolar fluid with $\mu=2$ in the absence of an electric field, $E=0$. Top panel, low shear rate $\gamma=1$ (shearthinning regime); Bottom panel, high shear rate $\gamma=6$ (around the onset of the shear-thickening regime). Notice that the grayscale for the shading is different for the top and bottom panels.

sion above for the configurational temperature actually amounts to being a working assumption, that is, an operational definition.

At the shear rates $\gamma=1$ and 6 , we set $\left(Q_{c}, \Delta t\right)$ $=(10,0.0005)$ and $(200,0.000025)$, respectively. All configurational thermostated simulations were run starting from an equilibrium $(\gamma=0)$ configuration for $200000 \Delta t$ at $\gamma \neq 0$; then, averages and standard deviations were estimated during a subsequent set of 20 blocks of 40000 time steps. Two system sizes were considered: $N=500$ and 1372 particles per central simulation cell. Figure 8 shows the pair distribution function in the $X Y$ plane for the simple dipolar fluid with $\mu=2$ at $\gamma=1$ and 6 where the electric field is turned off. Distortion away from spherical symmetry is evident at $\gamma=1$; this distortion is particularly pronounced at $\gamma=6$, which is around the onset of the shear-thickening regime of flow. For $N=1372$, the shear viscosity at $\gamma=6$ was found to be $\eta_{s}$ $=1.47 \pm 0.03$ for $\mu=0$ (Lennard-Jones fluid), $1.60 \pm 0.02$ for $\mu=2$ with $E=0$ (simple dipolar fluid, electric field off), and $1.52 \pm 0.03$ for $\mu=2$ with $E=1.5$ (simple dipolar fluid, electric field on). The system with $N=500$ yielded values of $\eta_{s}$ which differed by only \pm 0.02 from the values of $\eta_{s}$ for the system with $N=1372$. The shear viscosity of the simple dipolar fluid is higher than the corresponding Lennard-Jones (LJ) fluid at high shear rates: the first peak in the pair distribution function running along the compression axis, $g_{c}(r)$ (Fig. 9), is located at a smaller value of $r$ for the simple dipolar fluid than for the LJ fluid. The compression axis is

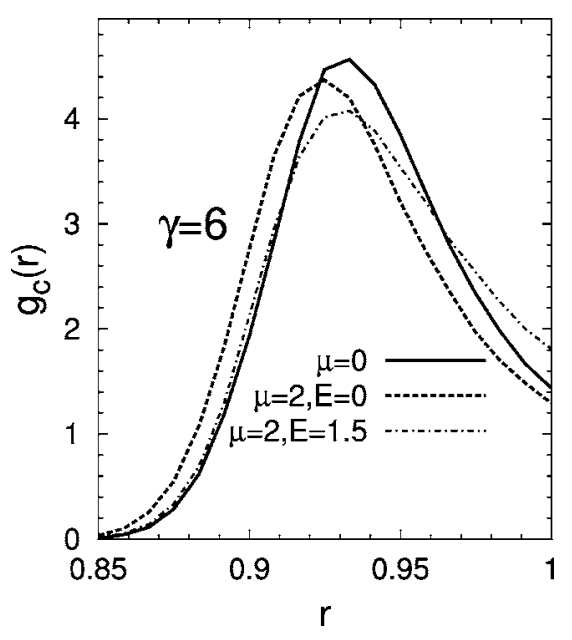

FIG. 9. Configurational thermostated SLLOD dynamics. Pair distribution function running along the compression axis, $g_{c}(r)$, at a shear rate of $\gamma=6$. Lennard-Jones fluid $(\mu=0)$ solid curve; simple dipolar fluid $(\mu=2)$ with no electric field, dashed curve; and simple dipolar fluid $(\mu=2)$ with electric field $(E=1.5)$, dot-dashed curve.

the direction along which the $g(\mathbf{r})$ appears most compressed; looking at Fig. 8, we see that this axis is located at an angle close to $135 \mathrm{deg}$ away from the $X$ axis in the $X Y$ plane. The pronounced compression at $\gamma=6$ reflects the existence of tight clusters of particles ${ }^{37,38}$ however, since the pair correlation function is derived from an average over all particles, that is, cluster particles and "spectator" particles, the $g(\mathbf{r})$ cannot be used as a measure of pair correlations pertaining to only the cluster particles. To see the cluster particles one needs to define a "cluster protocol" as discussed in Ref. 37 . An explicit or implicit solvent model was omitted from our simulations; therefore, the shear thickening generated at high shear rates must be a result of the jamming of particles within a cluster, and not a result of solvent effects such as the divergence of lubrication interactions or shear-induced depletion flocculation. ${ }^{12}$ This fact has already been discussed by Delhommelle and Petravic. ${ }^{37,38}$

If only the configurational degrees of freedom are thermostated under shear, then the temperature of the kinetic degrees of freedom, $T_{\text {kin,tr }}$, becomes greater than the target temperature; in addition, $T_{\text {kin,tr }}$ increases with increasing shear rate. For example, using only the translational configurational thermostat, Eqs. (10)-(12), $T_{\text {kin,tr }}$ was approximately three times larger than $T_{\text {conf,tr }}$ at a shear rate of $\gamma=6$. As a result, the shear viscosity of the simple dipolar fluid at high shear rates depended on the thermostatting mechanism used: the $\eta_{s}$ from a simulation using only the kinetic thermostated, profile-unbiased thermostat was higher than the $\eta_{s}$ from a simulation using only the translational configurational thermostated SLLOD dynamics (compare the values of $\eta_{s}$ from Fig. 12 to those stated in the paragraph above). Similar findings have also been reported by Travis and Braga in Ref. 59. In the statistical mechanics of highly nonequilibrium fluids under shear flow, there is no principle at present which states that the thermostatting of different sets of degrees of freedom is mutually exclusive: there is no reason for or against the thermostatting of some or all degrees of freedom, kinetic and configurational. We have not pursued this issue in this work. 
In addition, one could also choose to thermostat the orientational configurational degrees of freedom; ${ }^{34}$ we have not.

\section{Extended SLLOD dynamics}

One criticism of the kinetic thermostated, profileunbiased dynamics is that it results in a secondary flow profile, $\overline{\mathbf{u}}_{s}^{g}$, whose fluctuations vary in time at a rate comparable to those rates in the fluctuations of the thermal velocities, $\mathbf{p}_{i}^{\prime \prime}$. It has been stated by Bagchi et al. that this result is incorrect. They have modified the original SLLOD equations of motion, Eqs. (1) and (2), to create an "extended" SLLOD dynamics where the frequency in the fluctuations of $\overline{\mathbf{u}}_{s}^{g}$ can be controlled, ${ }^{43}$

$$
\begin{aligned}
& d \mathbf{r}_{i} / d t=\mathbf{p}_{i}^{\prime \prime} / m+\hat{\mathbf{e}}_{x} y_{i} \gamma+\mathbf{u}_{s i}, \\
& d \mathbf{p}_{i}^{\prime \prime} / d t=\mathbf{F}_{i}-\hat{\mathbf{e}}_{x} \gamma\left[p_{i y}^{\prime \prime}+m u_{s i y}\right]-m \mathbf{p}_{u i} / Q_{u}-\alpha^{T} \mathbf{p}_{i}^{\prime \prime}, \\
& \dot{\mathbf{u}}_{s i}=\mathbf{p}_{u i} / Q_{u}, \\
& \dot{\mathbf{p}}_{u i}=-\left[\mathbf{u}_{s i}-\overline{\mathbf{u}}_{s}^{g}\left(\mathbf{r}_{i}, t\right)\right]-\xi \mathbf{p}_{u i}, \\
& \dot{\xi}=\tau^{-2}\left[K_{u}\left(\mathbf{p}_{u}^{N}\right) / K_{u}^{0}-1\right] .
\end{aligned}
$$

Here, $K_{u}^{0}=3 N k_{B} T_{u}^{0} / 2$ and $K_{u}=\Sigma_{i} \mathbf{p}_{u i}^{2} /\left(2 Q_{u}\right)=\mathbf{p}_{u}^{N} \cdot \mathbf{p}_{u}^{N} /\left(2 Q_{u}\right)$, where $Q_{u}$ and $T_{u}^{0}$ are the secondary mass and temperature, respectively. The first two equations are simply the original SLLOD equations of motion, Eqs. (1) and (2), rewritten in terms of the thermal, peculiar velocity $\mathbf{p}_{i}^{\prime \prime}$, not in terms of $\mathbf{p}_{i}^{\prime}$ as in the original equations. Furthermore, in Eq. (2) the original secondary flow, $\overline{\mathbf{u}}_{s}^{g}$, is replaced by a new secondary flow, $\mathbf{u}_{s i}$. This "dynamical" secondary flow is treated as a extended phase-space variable with its own equations of motion, Eqs. (16) and (17): $\mathbf{u}_{s i}$ is kept close to the "guiding" secondary flow, $\overline{\mathbf{u}}_{s}^{g}$, via a simple harmonic potential, Eq. (17), and a Nosé-Hoover feedback mechanism, Eq. (18), with a multiplier, $\xi$. Equation (17) is essentially the equation of motion for a damped harmonic oscillator with friction $\xi$. The smaller the magnitude of $T_{u}$, the closer $\mathbf{u}_{s i}$ is to the guide $\overline{\mathbf{u}}_{s}^{g}$. The guiding secondary flow is still determined through the minimization of the residual, which incorporates a truncated Fourier series expansion for this flow. The temperature, $T_{u}^{0}$, in our simulations was set to 0.1 . The Nosé-Hoover parameter, $\tau$, controls how close the instantaneous kinetic energy, $K_{u}$, stays to the target kinetic energy, $K_{u}^{0}$; $\tau$ is an adjustable parameter that must be tuned. In our simulations $\tau$ was set to 0.01 ; deviations of the instantaneous secondary temperature, $T_{u}$, from the target of $T_{u}^{0}=0.1$ were less than $1 \%$.

The most important parameter for our purposes is the secondary mass $Q_{u}$. Given that the "spring constant" in Eq. (17) is set to 1 , increasing $Q_{u}$ should decrease the frequency in the oscillations of $\mathbf{u}_{s i}$, slowing the decay in the velocity time autocorrelation function of $\mathbf{u}_{s i}$. For the dynamical secondary flow, this function is

$$
C_{u s}(t)=\frac{\left\langle\mathbf{u}_{s}^{N}(t) \cdot \mathbf{u}_{s}^{N}(0)\right\rangle}{\left\langle\mathbf{u}_{s}^{N}(0) \cdot \mathbf{u}_{s}^{N}(0)\right\rangle},
$$

where

$$
\left\langle\mathbf{u}_{s}^{N}(t) \cdot \mathbf{u}_{s}^{N}(0)\right\rangle=\lim _{T \rightarrow \infty} \frac{1}{T} \int_{0}^{T} d \tau \frac{1}{N} \sum_{i=1}^{N} \mathbf{u}_{s i}(\tau+t) \cdot \mathbf{u}_{s i}(\tau) .
$$

Similarly, for the guiding secondary flow, we have

$$
C_{u g}(t)=\frac{\left\langle\mathbf{u}_{s}^{g}(t) \cdot \mathbf{u}_{s}^{g}(0)\right\rangle}{\left\langle\mathbf{u}_{s}^{g}(0) \cdot \mathbf{u}_{s}^{g}(0)\right\rangle},
$$

where

$$
\left\langle\mathbf{u}_{s}^{g}(t) \cdot \mathbf{u}_{s}^{g}(0)\right\rangle=\lim _{T \rightarrow \infty} \frac{1}{T} \int_{0}^{T} d \tau \frac{1}{N_{k}} \sum_{k=1}^{N_{k}} \mathbf{u}_{s}^{g}\left(\mathbf{q}_{k}, \tau+t\right) \cdot \mathbf{u}_{s}^{g}\left(\mathbf{q}_{k}, \tau\right) .
$$

Here, the simulation cell is divided into $6 \times 6 \times 6=216=N_{k}$ cubic subcells, where $\mathbf{q}_{k}$ refers to the position at the center of the subcell with index $k$.

To get an estimate for the value of $Q_{u}$, we consider the evolution of a harmonic oscillator with a spring constant set to 1: $Q_{u} d^{2} x / d t^{2}=-x$ where $x(t=0)=x_{0}$ and $d x_{0} / d t=0$. For the displacement variable, $x(t)$, we have simply $\langle x(t) x(0)\rangle / x_{0}^{2}$ $=0.5 \cos \left(t / \sqrt{Q_{u}}\right)$. From a kinetic thermostated, profileunbiased dynamics simulation of the simple dipolar fluid with $\mu=2$ and $E=0$, the normalized time autocorrelation function of the secondary flow at $\gamma=5.5$ decays to a value of $\sim 0.7$ after 0.03 units of time. Entering this simulation result into $\langle x(t) x(0)\rangle / x_{0}^{2}$ yields the estimate, $Q_{u} \sim 0.0014$.

In this section, we will examine the effects of varying the value of $Q_{u}$ on the $\mu=2$ dipolar fluid with the electric field turned off, $E=0$, and turned on, $E=1.5$. Our simulations start from an initial configuration that is the final configuration of a profile-unbiased dynamics simulation performed at $\gamma=5.5$; at this shear rate and for this dynamics, the fluid exhibits the onset on shear thickening. Given this initial configuration, the extended SLLOD dynamics simulations were run for 180000 time steps of size $\Delta t=0.0005$. Averages were then collected for at least another $200000 \Delta t$, and standard deviations were calculated by dividing these into blocks of size $20000 \Delta t$.

Figure 10 shows the effect of varying $Q_{u}$ on the decay of $C_{u s}(t)$ and $C_{u g}(t)$ at $\gamma=5.5$ with $E=0$. Clearly, as $Q_{u}$ increases, starting from a value of $Q_{u}=0.001$, the rate of decay of these time autocorrelation functions decreases; we expected such a dependence on $Q_{u}$ because the extended dynamics was constructed to produce this dependence. Again, the dynamical secondary flow, $\mathbf{u}_{s i}$, is entered into the SLLOD dynamics, and this flow is forced by the extended SLLOD dynamics to follow the guiding secondary flow, $\overline{\mathbf{u}}_{s}^{g}$. However, $\overline{\mathbf{u}}_{s}^{g}$ is affected by changes in the fluid structure and dynamics, which in turn are affected by the $\overline{\mathbf{u}}_{s i}$; consequently, $\overline{\mathbf{u}}_{s}^{g}$ indirectly depends on the dynamics of the extended secondary flow. In short, the two secondary flows should be mutually dependent. It is satisfying to see that the decays in these two flows are consistent, as one would hope to see as confirmation that the extended dynamics was being integrated correctly.

As noted in the caption of Fig. 10, the shear viscosity, $\eta_{s}$, decreases for increasing $Q_{u}$ at $\gamma=5.5$ with $E=0$. The structure of the fluid at $Q_{u}=0.1$, the highest value of $Q_{u}$ 


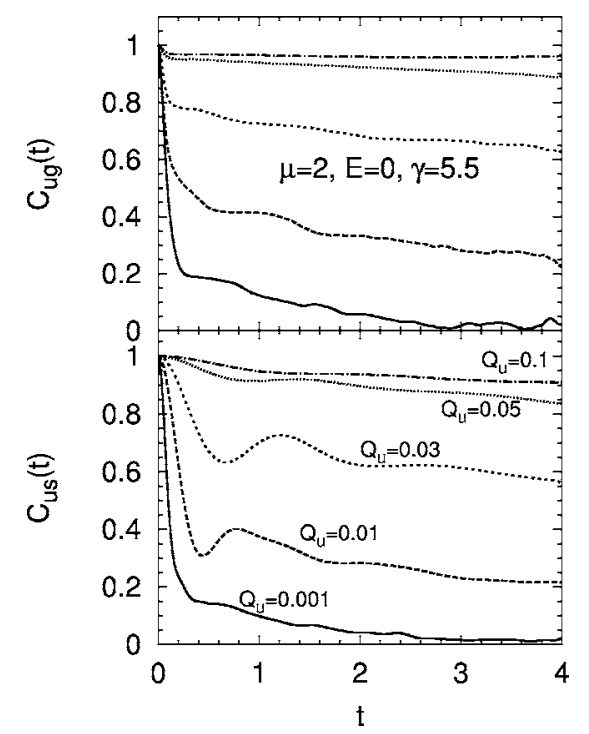

FIG. 10. Extended SLLOD dynamics. Normalized time autocorrelations functions for the guiding flow, $\overline{\mathbf{u}}_{s}^{g}(\mathbf{r})$ (top panel), and the dynamical secondary flow, $\mathbf{u}_{s i}$ (bottom panel). The "secondary mass," $Q_{u}$, is varied, and the electric field is turned off. The associated shear viscosities are as follows: $\left(Q_{u}=0.001, \eta_{s}=1.83 \pm 0.03\right), \quad\left(Q_{u}=0.01, \eta_{s}=1.54 \pm 0.08\right), \quad\left(Q_{u}=0.03, \eta_{s}\right.$ $=1.27 \pm 0.08),\left(Q_{u}=0.05, \eta_{s}=0.96 \pm 0.27\right)$, and $\left(Q_{u}=0.1, \eta_{s}=1.04 \pm 0.04\right)$.

examined, is a mixture of strings aligned along the flow direction with hexagonal symmetry and some particles organized in a more disorganized, amorphous conformation. To observe a further drop in $\eta_{s}$ at $Q_{u}=0.1$, we need to increase the shear rate above $\gamma=5.5$.

We now apply an electric field $(E=1.5)$ directed along the $Z$ axis and repeat the experiment of varying $Q_{u}$ at a fixed shear rate of $\gamma=5.5$, measuring the shear viscosity. With the electric field turned on, $\eta_{s}$ decreases faster with increasing $Q_{u}$ at fixed $\gamma$ than with the electric field turned off (see the top panel of Fig. 11). Alternatively, we can fix $Q_{u}$ and vary $\gamma$

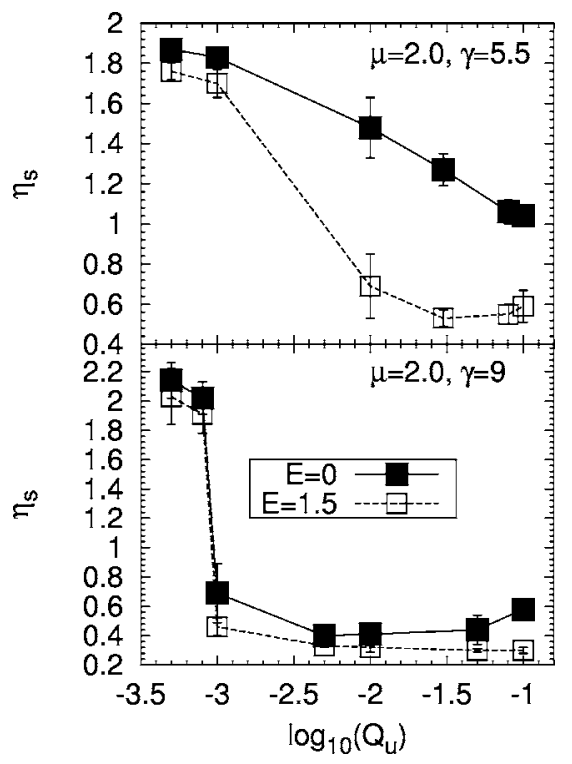

FIG. 11. Extended SLLOD dynamics. Shear viscosity, $\eta_{s}=-P_{y x} / \gamma$, as a function of the logarithm to the base 10 of the secondary mass, $Q_{u}$, for a simple dipolar fluid with $\mu=2$. The shear rate is $\gamma=5.5$ (top panel) and 9 (bottom panel). The electric field is turned off $(E=0)$, and turned on $(E$ $=1.5)$.

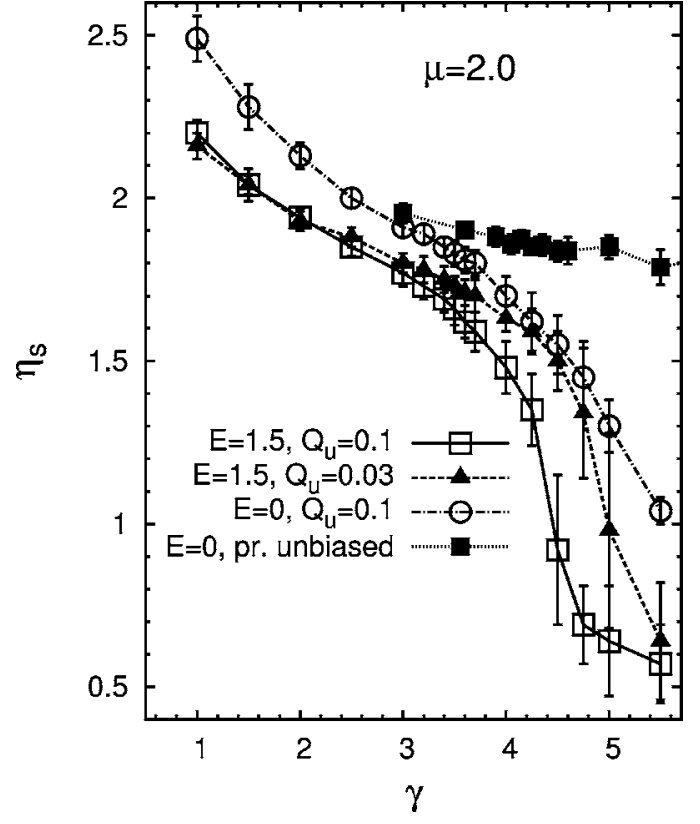

FIG. 12. Extended SLLOD and kinetic thermostated, profile-unbiased dynamics. Shear viscosity, $\eta_{s}=-P_{y x} / \gamma$, as a function of shear rate, $\gamma$, for the simple dipolar fluid with $\mu=2$ at two values of the electric field intensity, $E$. The secondary mass, $Q_{u}$, is varied.

measuring $\eta_{s}$. We observe two effects (Fig. 12): for $E=0$ and 1.5, as $Q_{u}$ is increased the transition to a string phase begins at a smaller value of shear rate; in addition, for a given $Q_{u}$, this transition begins at a smaller $\gamma$ for $E=1.5$ than for $E$ $=0$. This latter effect is reminiscent of the subcritical transition to a string phase observed when applying a suitably directly electric field while using the kinetic thermostated, profile-biased dynamics (Sec. IV A).

Next, we consider the decay of the time autocorrelation functions for the guiding secondary flow, $\mathbf{u}_{s}^{g}$, and the peculiar, thermal velocity, $\mathbf{p}_{i}^{\prime \prime}$, at $\gamma=5.5$ and $Q_{u}=0.03\left[\log _{10}\left(Q_{u}\right)\right.$ $=-1.5]$. For both $E=1.5$ and $E=0$, the implementation of the extended dynamics has the desired effect of slowing the fluctuations in $\mathbf{u}_{s}^{g}$ relative to the frequency of the fluctuations of the thermal velocities. In addition, by applying an electric field at these values of $Q_{u}$ and $\gamma$, the decay of the time autocorrelation function for the thermal velocities, $C_{v}(t)$ (see the top panel of Fig. 13) displays a deeper initial well. This function is calculated as follows:

$$
C_{v}(t)=\frac{\left\langle\mathbf{p}^{\prime \prime}(t) \cdot \mathbf{p}^{\prime \prime}(0)\right\rangle}{\left\langle\mathbf{p}^{\prime \prime}(0) \cdot \mathbf{p}^{\prime \prime}(0)\right\rangle},
$$

where

$$
\left\langle\mathbf{p}^{\prime \prime}(t) \cdot \mathbf{p}^{\prime \prime}(0)\right\rangle=\lim _{T \rightarrow \infty} \frac{1}{T} \int_{0}^{T} d \tau \frac{1}{N} \sum_{i=1}^{N} \mathbf{p}_{i}^{\prime \prime}(t+\tau) \cdot \mathbf{p}_{i}^{\prime \prime}(t) .
$$

The increased well depth upon going from $E=0$ to $E=1.5$ reflects an enhanced caging effect consistent with the formation of a strong, homogeneous string phase when $E=1.5$.

The extended SLLOD dynamics controls the frequency of the fluctuations in the secondary flows; if these fluctuations are sufficiently slowed, then a string phase forms as evidenced by the large decrease in shear viscosity, $\eta_{s}$. How- 


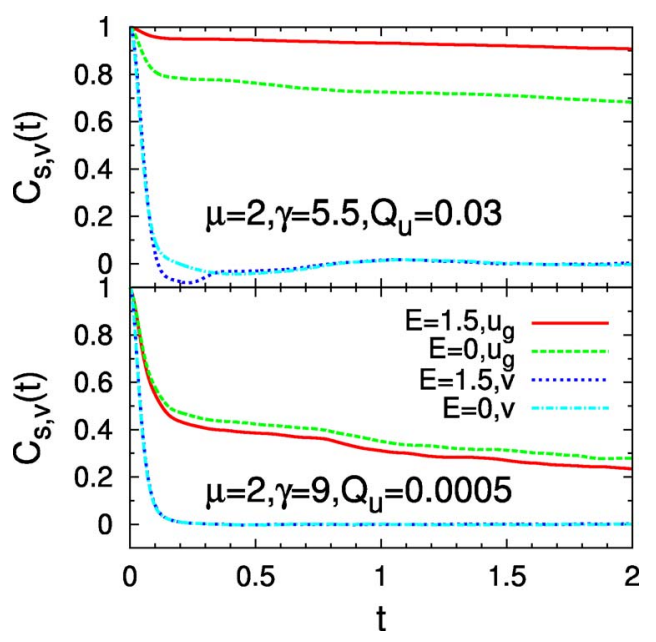

FIG. 13. (Color online) Extended SLLOD dynamics. Time autocorrelation functions for the thermal velocities, $\mathbf{p}^{\prime \prime}$ (labeled $v$ ), and the guiding, secondary flow, $\mathbf{u}_{s}^{g}(\mathbf{r})$ (labeled $u_{g}$ ), at high (top panel, $\gamma=5.5$ ) and very high (bottom panel, $\gamma=9$ ) shear rates. Refer to Fig. 11. In each panel, the upper two curves are associated with $u_{g}$, while the lower two are associated with $v$.

ever, for colloidal suspensions there is no experimental evidence verifying the existence of the string phase. If there is any merit to the criticism stated by Bagchi et al. against the kinetic thermostated, profile-unbiased dynamics, then the extended SLLOD dynamics still can be used to slow the fluctuations in the secondary flows, so that the distinction between secondary flow and thermal fluctuations is made, while also not generating a string phase. What then remains is to ask whether a string phase, which is not present in the absence of the electric field, can be generated/stabilized in the presence of a suitably directed electric field. If $Q_{u}$ is decreased, does there exist a $Q_{u}$ where (1) with $E \neq 0, \eta_{s}$ is very low, consistent with the existence of a string phase, but (2) with $E=0, \eta_{s}$ is still high, consistent with the experimental finding of the nonexistence of a string phase? We have already demonstrated that such a $Q_{u}$ exists at $\gamma=5.5$ (see the top panel of Fig. 11). If we can find a $Q_{u}$ where this scenario is true at all $\gamma$, then we will have provided some evidence for the possible existence of the string phase (for a simple dipolar fluid under shear flow) stabilized by external means.

At $\gamma=9$ (a very high shear rate), we cannot find such a $Q_{u}$; in fact, the sudden drop in $\eta_{s}$ with increasing $Q_{u}$ occurs at approximately the same value of $Q_{u}$ for $E=0$ as for $E$ $=1.5$ (see the bottom panel of Fig. 11). Here, the simulation runs were started from an initial configuration that was the final configuration of a kinetic thermostated, profile-unbiased dynamics simulation at $\gamma=9$; given this configuration, extended SLLOD dynamics simulations were run for 180000 $\Delta t$ of $\Delta t=0.0005$; averages and standard deviations were calculated then during a subsequent 10 blocks of $20000 \Delta t$. This finding suggests that the string phase, at least in the colloidal regime ${ }^{12}$ for an unbounded fluid (a simulation cell with no confining walls), does not exist at very high shear rates, rather a shear-thickening phase exists. Examining the thermal time autocorrelation functions at $Q_{u}=0.0005$, a value of $Q_{u}$ immediately before the $Q_{u}$ where the $\eta_{s}$ drops rapidly in value with increasing $Q_{u}$ at $\gamma=9$, we see no caging effect (see the bottom panel of Fig. 13), just a simple monotonic decay, and, as desired, the fluctuations in the secondary flows, $\mathbf{u}_{s}^{g}$, are slower than those fluctuations in the thermal velocities.

\section{SUMMARY AND CONCLUSIONS}

We have explored the possibility of electric field assisted phase transitions of a simple dipolar fluid under strong shear flows. The results depended heavily on the thermostat used to extract heat from the fluid. A profile-biased thermostat, which assumes a linear streaming flow profile with no secondary flow, produces a string phase at high shear rates. Applying an electric field, directed perpendicular to both the shear flow and the shear gradient directions, enhances pair correlations along the field direction, leading to the start of the transition to the string phase occurring at lower shear rates. In other words, given the existence of the string phase, the string phase can be stabilized by an electric field at "subcritical" shear rates. However, after switching to a kinetic thermostated, profile-unbiased thermostat, the string phase disappears, as do the subcritical transitions assisted by the suitably directed electric field.

There has been one criticism given against the use of the kinetic thermostated, profile-unbiased thermostat: it produces fluctuations in the secondary flows which are as fast as those pertaining to the thermal velocities. Implementing the extended SLLOD dynamics put forth by Bagchi and co-workers, ${ }^{43}$ which introduces a "dynamical" secondary flow with its own equation of motion and associated "secondary" mass, solves this problem. In addition, if the fluctuations in the secondary flows are sufficiently slowed, then we can regain the string phase at high shear rates. Given this string phase, applying an electric field has the same effect on the value of the critical shear rate needed for the start of the transition to the string phase: the critical shear rate is lowered. The existence of string phases for an unconfined colloidal suspension has not been clearly observed experimentally. ${ }^{10,11}$ In addition, recent "atomic" simulations (atomic in the sense that the hydrodynamic flow effects of the solvent and Brownian forces are not included in the simulation dynamics), which employ a configurational thermostat, have also not yielded a string phase, but rather a shear-thickening phase characterized by the formation of densely packed clusters of particles. ${ }^{26,37}$ Given these findings, we tried to determine whether applying an electric field could still stabilize a string phase at high shear rates given a shear-thickening phase in the absence of an electric field where the fluctuations in the secondary flows are slowed with respect to the thermal fluctuations. We found that in general the electric field did not have this stabilizing effect. In the shear-thickening regime, the shear viscosity of the simple dipolar fluid was higher than that of the LennardJones fluid: dipolar interactions enhance the jamming effect between particles at very high shear rates; this enhanced jamming was lessened by the application of the electric field in the $Z$ direction. The only general effect found at all shear rates of applying this field along the $Z$ direction, regardless of the thermostated dynamics used, was a small reduction in the shear viscosity of the simple dipolar fluid and not the 
radical drop in shear viscosity seen during the formation of the string phase. We did not characterize the full electroviscous (magnetoviscous) response of our dipolar fluid to a uniform electric (magnetic) field oriented parallel or perpendicular to the flow; however, we do feel that determining and understanding this response for a dense fluid at high shear rates would be of great general interest.

Despite the seeming nonexistence of the string phase, our work here is not without merit. Of course, we have shown that the shear-thickening phase, characterized by no long-range, strong spatial correlations, is stable against the application of an electric field along the $Z$ direction. Although the string phase is not a flow structure existing at high shear rates in the colloidal regime, ${ }^{12}$ a recent simulation study $^{13}$ has predicted that it might be a structure found at high shear rates for a noncolloidal suspension. The simulations here employed Stokesian dynamics to generate implicitly the hydrodynamics of the suspending solvent; these dynamics can be contrasted with other methods such as smooth particle hydrodynamics (SPH) (Ref. 60) or multiparticle collision (MPC) dynamics, ${ }^{61-64}$ which generate explicitly hydrodynamic effects by using an explicit solvent particle model. In the absence of electrostatic pair interactions, strong layering transitions have been observed in simulations of confined model colloidal suspensions under steady shear flows $^{64}$ and in experiments of colloidal suspensions under oscillating shear flows. ${ }^{9,29,65}$ Some of the insights and physical intuition gained from this study should be pertinent toward the future understanding of confined dipolar fluids ${ }^{66,67}$ under steady shear flows, as well as confined or effectively unbounded dipolar fluids under time-dependent, oscillating shear flows.

\section{ACKNOWLEDGMENTS}

The financial support of the Natural Sciences and Engineering Research Council of Canada (NSERC) is gratefully acknowledged. The author would also like to thank Professor Gren N. Patey for his helpful comments made at the start of this work.

\footnotetext{
${ }^{1}$ D. Evans and R. Watts, Chem. Phys. 48, 321 (1980).

${ }^{2}$ S. Hess, Phys. Rev. A 22, 2844 (1980).

${ }^{3}$ D. Heyes, J. Kim, C. Montrose, and T. Litovitz, J. Chem. Phys. 73, 3987 (1980).

${ }^{4}$ H. Hanley, D. Evans, and S. Hess, J. Chem. Phys. 78, 1440 (1983).

${ }^{5}$ H. Hanley, J. Rainwater, N. Clarke, and B. Ackerson, J. Chem. Phys. 79 4448 (1983)

${ }^{6}$ D. Evans, H. Hanley, and S. Hess, Phys. Today 27, 26 (1984).

${ }^{7}$ D. Heyes, J. Chem. Phys. 85, 997 (1986).

${ }^{8}$ D. Heyes, J. Chem. Soc., Faraday Trans. 2 82, 1365 (1986).

${ }^{9}$ B. J. Ackerson and P. N. Pusey, Phys. Rev. Lett. 61, 1033 (1988).

${ }^{10}$ B. J. Ackerson, J. Rheol. 34, 553 (1990).

${ }^{11}$ B. J. Ackerson, Physica A 174, 15 (1991).

${ }^{12}$ J. F. Brady, Chem. Eng. Sci. 56, 2921 (2001).

${ }^{13}$ A. Sierou and J. F. Brady, J. Rheol. 46, 1031 (2002).

${ }^{14}$ M. Newstein, H. Wang, N. P. Balsara, A. A. Lefebvre, Y. Shnidman, H. Watanabe, K. Osaki, T. Shikata, H. Niwa, and Y. Morishima, J. Chem. Phys. 111, 4827 (1999)

${ }^{15}$ B. Maranzano and N. J. Wagner, J. Chem. Phys. 117, 10291 (2002).
}

${ }^{16}$ M. P. Allen and D. J. Tildesley, Computer Simulation of Liquids (Clarendon, Oxford, 1987).

${ }^{17}$ D. Evans and G. Morriss, Statistical Mechanics of Nonequilibrium Liquids (Academic, London, 1990).

${ }^{18}$ A. Lees and S. Edwards, J. Phys. C 5, 1921 (1972).

${ }^{19}$ D. Evans and G. Morriss, Phys. Rev. Lett. 56, 2172 (1986).

${ }^{20}$ K. Travis, P. Daivis, and D. Evans, J. Chem. Phys. 103, 1109 (1995).

${ }^{21}$ K. Travis, P. Daivis, and D. Evans, J. Chem. Phys. 103, 10638 (1995).

${ }^{22}$ K. Travis, P. Daivis, and D. Evans, J. Chem. Phys. 105, 3893 (1996).

${ }^{23}$ X.-F. Yuan and M. Allen, Physica 240A, 145 (1997).

${ }^{24}$ W. Loose and S. Hess, Rheol. Acta. 28, 91 (1989).

${ }^{25}$ W. Loose and G. Ciccotti, Phys. Rev. A 45, 3859 (1992).

${ }^{26}$ J. Delhommelle, J. Petravic, and D. J. Evans, Phys. Rev. E 68, 031201 (2003).

${ }^{27}$ J. Delhommelle, J. Petravic, and D. J. Evans, J. Chem. Phys. 119, 11005 (2003).

${ }^{28}$ J. Delhommelle, J. Petravic, and D. J. Evans, J. Chem. Phys. 120, 6117 (2004).

${ }^{29}$ J. Delhommelle, Phys. Rev. B 69, 144117 (2004).

${ }^{30}$ J. Delhommelle and J. Petravic, J. Chem. Phys. 118, 2783 (2003).

${ }^{31}$ C. Braga and K. P. Travis, J. Chem. Phys. 123, 134101 (2005).

${ }^{32}$ H. H. Rugh, Phys. Rev. Lett. 78, 772 (1997).

${ }^{33}$ O. G. Jepps, G. Ayton, and D. J. Evans, Phys. Rev. E 62, 4757 (2000).

${ }^{34}$ A. A. Chialvo, J. M. Simonson, P. T. Cummings, and P. G. Kusalik, J. Chem. Phys. 114, 6514 (2001).

${ }^{35}$ J. Erpenbeck, Phys. Rev. Lett. 52, 1333 (1984).

${ }^{36}$ J. McWhirter and G. Patey, J. Chem. Phys. 117, 2747 (2002).

${ }^{37}$ J. Delhommelle, Eur. Phys. J. E 15, 65 (2004).

${ }^{38}$ J. Delhommelle and J. Petravic, J. Chem. Phys. 123, 074707 (2005).

${ }^{39}$ R. Rosensweig, Ferrohydrodynamics (Cambridge University Press, Cambridge, 1985).

${ }^{40}$ E. Blums, A. Cebers, and M. Maiorov, Magnetic Fluids (de Gruyter, New York, 1997).

${ }^{41}$ H. Mamiya, I. Nakatani, and T. Furubayashi, Phys. Rev. Lett. 84, 6106 (2000).

${ }^{42}$ J. F. Brady, Annu. Rev. Fluid Mech. 20, 111 (1988).

${ }^{43}$ K. Bagchi, S. Balasubramanian, C. Mundy, and M. Klein, J. Chem. Phys. 105, 11183 (1996)

${ }^{44}$ C. Saluena, A. Perez-Madrid, and J. Rubi, J. Chem. Phys. 96, 6950 (1992).

${ }^{45}$ C. Saluena, A. Perez-Madrid, and J. Rubi, J. Colloid Interface Sci. 164, 269 (1994).

${ }^{46}$ W. F. Hall and S. N. Busenberg, J. Chem. Phys. 51, 137 (1969).

${ }^{47}$ J. P. McTague, J. Chem. Phys. 51, 133 (1969).

${ }^{48}$ M. I. Shliomis, Sov. Phys. Usp. 17, 153 (1974).

${ }^{49}$ M. Kröger, P. Ilg, and S. Hess, J. Phys.: Condens. Matter 15, S1403 (2003).

${ }^{50}$ P. Ilg and S. Hess, Z. Naturforsch. A: Phys. Sci. 58A, 589 (2003).

${ }^{51}$ J. McWhirter, J. Chem. Phys. 118, 2824 (2003).

${ }^{52}$ D. Evans, Mol. Phys. 32, 1171 (1976).

${ }^{53}$ S. D. Leeuw, J. Perram, and E. Smith, Proc. R. Soc. London, Ser. A 373, 27 (1980).

${ }^{54}$ S. D. Leeuw, J. Perram, and E. Smith, Proc. R. Soc. London, Ser. A 373, 57 (1980).

${ }^{55}$ P. Kusalik, J. Chem. Phys. 93, 3520 (1990).

${ }^{56}$ D. R. Wheeler, N. G. Fuller, and R. Rowley, Mol. Phys. 92, 55 (1997).

${ }^{57}$ R. Eppenga and D. Frenkel, Mol. Phys. 52, 1303 (1984).

${ }^{58}$ Y. V. Kalyuzhnyi, S. T. Cui, and H. D. Cochran, Phys. Rev. E 63, 011209 (2000).

${ }^{59}$ K. P. Travis and C. Braga, Mol. Phys. 104, 3735 (2006).

${ }^{60}$ J. J. Monaghan, J. Comput. Phys. 110, 399 (1994).

${ }^{61}$ A. Malevanets and R. Kapral, J. Chem. Phys. 110, 8605 (1999).

${ }^{62}$ A. Malevanets and R. Kapral, J. Chem. Phys. 112, 7260 (2000).

${ }^{63}$ M. Ripoll, K. Mussawisade, R. G. Winkler, and G. Gompper, Phys. Rev. E 72, 016701 (2005).

${ }^{64}$ M. Hecht, J. Harting, and H. J. Herrmann, Phys. Rev. E 75, 051404 (2007).

${ }^{65}$ I. Cohen, T. Mason, and D. Weitz, Phys. Rev. Lett. 93, 046001 (2004).

${ }^{66}$ S. H. L. Klapp and M. Schoen, J. Chem. Phys. 117, 8050 (2002).

${ }^{67}$ V. A. Froltsov and S. H. L. Klapp, J. Chem. Phys. 124, 134701 (2006). 